\section{The birds of Araku, Visakhapatnam, Andhra Pradesh, India}

\section{T. Selva Kumar ${ }^{1}$, Rachna Chandra ${ }^{2}$ \& P.A. Azeez ${ }^{1}$ \\ 1,2 Environmental Impact Assessment Division, Sálim Ali Centre for Ornithology and Natural History (SACON), Anaikatty (PO), Coimbatore, Tamil Nadu 641108, India \\ Email: ${ }^{1}$ selvakt@ gmail.com, ${ }^{2}$ rachnaeia @ gmail.com (corresponding author), 1 azeezpa@gmail.com}

Documentation of the avifauna in Andhra Pradesh has been undertaken by several authors since early $20^{\text {th }}$ century by the likes of Whistler \& Kinnear (1930-37), Abdulali (1945, 1953), Raju \& Price (1973), Price (1978, 1979, 1990), Ripley et al. (1987-88), and Srinivasulu (2004, 2006). Some of the notable works that covered the distribution, status and habitat of birds in the Visakhapatnam Hills were that of Raju \& Selvin (1971) and Raju \& Price (1973). In 1944, Abdulali (1945) collated a list of the 'Birds of Vizagapatam' that included 277 species from Anantagiri, Sankrametta and Lammasinghi. Ripley et al. (1987-88) in an extensive survey covering Lammasinghi, Valaspara, Sapparla, Bhadrachalam, Pedevalasa, Joythimamidi, Wangasara, lankapakalu and Anantagiri reported 160 species of birds. However, detailed surveys on the avifauna of Araku Valley appear scarce. In recent years some of the hill ranges of this area and their neighbourhoods have been identified as potential sites for mining bauxite. In view of habitat degradation that is likely to happen with mining activities, it was felt vital to acquire baseline data on the biodiversity of the region; an attempt is made here to compile and categorize the avifauna of Araku hills and valley based on field surveys from December 2006 to September 2007.

\section{Materials and Methods}

Araku Valley (Fig. 1) forms an integral part of Ananthagiri Hills of the Eastern Ghats in Visakhapatnam District of Andhra Pradesh. The Araku Valley (18012'34"-18 ${ }^{\circ} 25^{\prime} 12^{\prime \prime} \mathrm{N}$ \& $82^{\circ} 51^{\prime} 40^{\prime \prime}-83^{\circ} 06^{\prime} 53^{\prime \prime} E$ ), spread over an area of $36 \mathrm{~km}^{2}$, is characterized by undulating terrain, valleys and plateaus at

Date of publication (online): 26 January 2010

Date of publication (print): 26 January 2010

ISSN $0974-7907$ (online) | 0974-7893 (print)

Editor: J. Praveen

Manuscript details:

Ms \# 02108

Received 16 December 2008

Final received 28 September 2009

Finally accepted 01 December 2009

Citation: Kumar, T.S., R. Chandra \& P.A. Azeez (2010). The birds of Araku, Visakhapatnam, Andhra Pradesh, India. Journal of Threatened Taxa 2(1): 662665.

Copyright: () T. Selva Kumar, Rachna Chandra \& P.A. Azeez 2010. Creative Commons Attribution 3.0 Unported License. JoTT allows unrestricted use of this article in any medium for non-profit purposes, reproduction and distribution by providing adequate credit to the authors and the source of publication.

Acknowledgments: The authors are thankful to the locals of the region for their assistance during the field surveys. We are also grateful to Mr. S. Kaliraj, for his help and co-operation. We also thank our colleagues for support.

OPEN AGCESS | FREE DOWNLOAD
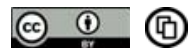

the hilltops. The area has huge deposits of bauxite mostly on the hilltops (Chandra et al. 2007; Azeez et al. 2008) predominantly covered with lateritic soil (bauxite capping over khondalite). The vegetation in the area is of moist mixed deciduous forest and dry savannah forest types intermingled with scrub (Champion \& Seth 1968). Apparently, forests in most of the area are secondary in nature, due to the extensive shifting or slash and burn cultivation (podu) that is in practice in the area since long. The altitude of the area ranges from $600-1600 \mathrm{~m}$ and the climate is very cold during winter (November to January) and warm during summer (March to May) with temperature varying from $10^{\circ} \mathrm{C}$ to $37.8^{\circ} \mathrm{C}$. The annual rainfall in Araku Valley ranges between $800-1000 \mathrm{~mm}$ in the plains and $1000-2000 \mathrm{~mm}$ in the hills. The Araku Valley mandal has a total of 11,608 households with a rural population of 49,619 and 6,340 urban. The rural population of Araku comprises mainly of indigenous tribes such as Bhagata, Khond, Konda Reddi, Samantha and other communities which come under Schedule $\mathrm{V}$ of Scheduled Tribes and other Traditional Forest Dwellers (Recognition of Forest Rights) Rules 2007. They largely depend on local forest and natural resources for livelihood. Phoenix sp. dominating the hill-top vegetation is extensively used for several purposes by the locals. Several other plants in the area are important for the tribes and are exploited for timber, tubers, fodder and fuel-wood. Hunting for small forest animals and birds is a traditional custom among the indigenous people and probably a means of supplementary animal protein.

Birds were recorded by both direct sighting and calls. Field surveys were carried out during December 2006 to September 2007 by systematically walking along transects laid appropriately to representatively cut through the study area. The variable width line-transect method (Bibby et al. 1993) was adopted for the survey. In the three major hillocks (Raktakonda, Galikonda and Chittamgondi) identified for bauxite mining, eight variable width line-transects each of one kilometer length were laid in each site. Transects traversed agriculture land, silver oak plantation, scrub jungle, well wooded forest and Phoenix dominated plateaus. Opportunistic surveys in the nearby villages, valleys and plains were also done to document a comprehensive checklist of birds of the area. The birds sighted during the period were grouped into breeding birds or residents (B), local migrants (LM), and migrants (M). These categorizations were done largely based on Ali \& Ripley (1983), Ali \& Ripley (1969) and Grimmett et al. (1999).

\section{Results}

A total of 147 species of birds falling within 105 genera and 43 families were recorded during the study (Table 1). The birds in Araku Valley include four globally threatened species (BirdLife International 2001), namely - the Purple Wood-Pigeon Columba punicea, Greater Spotted Eagle Aquila clanga, Lesser Kestrel Falco naumanni and Pallid Harrier Circus macrourus (Table 2). Eleven species of owls were recorded from the area, of which four species built nest in scrub jungle. The 11 owl species have been recorded from the area by the earlier researches. The frequently sighted bird families and subfamilies were Pycnonotidae (bulbuls), Sylviinae (warblers), Muscicapidae (flycatchers) and Turdinae (babblers). Speckled Piculet Picumnus innominatus, Rufous Woodpecker Celeus brachyurus, Brown-headed Barbet Megalaima zeylanica, 
Table 1. Birds observed in the environs of Araku Valley (classification as per Manakadan \& Pittie 2004)

\begin{tabular}{l} 
Common name \\
\hline Ardeidae \\
Little Egret \\
Median Egret \\
Cattle Egret \\
Indian Pond-Heron \\
Accipitridae \\
Oriental Honey-Buzzard \\
Black-shouldered Kite \\
Black Kite \\
Brahminy Kite \\
Crested Serpent-Eagle \\
Pallid Harrier \\
Montagu's Harrier \\
Shikra \\
White-eyed Buzzard \\
Black Eagle \\
Tawny Eagle \\
Changeable Hawk-Eagle
\end{tabular}

\section{Falconidae}

Lesser Kestrel

\section{Phasianidae}

Painted Francolin

Rain Quail

Red Spurfowl

Painted Spurfowl

Red JungleFowl

Grey Junglefowl

Indian Peafowl

\section{Columbidae}

Blue Rock Pigeon

Purple Wood-Pigeon

Little Brown Dove

Spotted Dove

Eurasian Collared-Dove

Emerald Dove

Green Imperial-Pigeon

Psittacidae

Rose-ringed Parakeet

Cuculidae

Brainfever Bird

Indian Cuckoo

Indian Plaintive Cuckoo

Drongo Cuckoo

Asian Koel

Small Green-billed Malkoha

Greater Coucal

\section{Tytonidae}

Barn Owl

\section{Strigidae}

Oriental Scops-Owl

Collared Scops-Owl

Eurasian Eagle-Owl

Brown Fish-Owl

Mottled Wood-Owl

Brown Wood-Owl

Jungle Owlet

Spotted Owlet

Brown Hawk-Owl

Short-eared Owl

Caprimulgidae

Common Indian Nightjar

Franklin's Nightjar

Apodidae

Asian Palm-Swift

House Swift

Trogonidae

Malabar Trogon

Scientific name

Status

Egretta garzetta (Linnaeus, 1766)

Mesophoyx intermedia (Wagler, 1829)

Bubulcus ibis (Linnaeus, 1758)

Ardeola grayii (Sykes, 1832)

Pernis ptilorhynchus (Temminck, 1821)

Elanus caeruleus (Desfontaines, 1789)

Milvus migrans (Boddaert, 1783)

Haliastur indus (Boddaert, 1783)

Spilornis cheela (Latham, 1790

Circus macrourus (S.G. Gmelin, 1770)

Circus pygargus (Linnaeus, 1758)

Accipiter badius (Gmelin, 1788)

Butastur teesa (Franklin, 1832)

Ictinaetus malayensis (Temminck, 1822)

Aquila rapax (Temminck, 1828)

Spizaetus cirrhatus (Gmelin, 1788)

Falco naumanni Fleischer, 1818

Francolinus pictus (Jardine \& Selby, 1828) Coturnix coromandelica (Gmelin, 1789)

Galloperdix spadicea (Gmelin, 1789)

Galloperdix lunulata (Valenciennes, 1825)

Gallus gallus (Linnaeus, 1758)

Gallus sonneratii Temminck, 1813

Pavo cristatus Linnaeus, 1758

Columba livia Gmelin, 1789

Columba punicea Blyth, 184

Streptopelia senegalensis (Linnaeus, 1766)

Streptopelia chinensis (Scopoli, 1786)

Streptopelia decaocto (Frivaldszky, 1838)

Chalcophaps indica (Linnaeus, 1758)

Ducula aenea (Linnaeus, 1766)

Psittacula krameri (Scopoli, 1769)

Hierococcyx varius (Vahl, 1797)

Cuculus micropterus Gould, 1838

Cacomantis passerinus (Vahl, 1797)

Surniculus lugubris (Horsfield, 1821)

Eudynamys scolopacea (Linnaeus, 1758)

Phaenicophaeus viridirostris (Jerdon, 1840)

Centropus sinensis (Stephens, 1815)

Tyto alba (Scopoli, 1769)

Otus sunia (Hodgson, 1836)

Otus bakkamoena Pennant, 1769

Bubo bubo (Linnaeus, 1758)

Ketupa zeylonensis (Gmelin, 1788)

Strix ocellata (Lesson, 1839)

Strix leptogrammica Temminck, 1831

Glaucidium radiatum (Tickell, 1833)

Athene brama (Temminck, 1821)

Ninox scutulata (Raffles, 1822)

Asio flammeus (Pontoppidan, 1763)

Caprimulgus asiaticus Latham, 1790

Caprimulgus affinis Horsfield, 1821

Cypsiurus balasiensis (J.E. Gray, 1829)

Apus affinis (J.E. Gray, 1830)

Harpactes fasciatus (Pennant, 1769)
B

B

B

B

B

B

B

WV

WV

B

B

BM

DM

Bufous Woodpecker

Woodpecker

PM Pittidae

Indian Pitta

Alaudidae

Rufous-tailed Finch-Lark

Hirundinidae

Red-rumped Swallow

Motacillidae

Large Pied Wagtail

Yellow Wagtail

Grey Wagtail

Paddyfield Pipit

Eurasian Tree Pipit

Campephagidae

Large Cuckoo-Shrike

Black-headed Cuckoo-Shrike

Small Minivet

White-bellied Minivet

Scarlet Minivet

Common Woodshrike

Pycnonotidae

Black-crested Bulbul

Red-whiskered Bulbul

Red-vented Bulbul

Irenidae

Common Iora

Jerdon's Chloropsis

Gold-fronted Chloropsis

B

aniidae

Brown Shrike

Bay-backed Shrike

LM Rufous-backed Shrike

Turdinae

Blue-headed Rock-Thrush

Blue Rock-Thrush

Orange-headed Thrush

Tickell's Thrush

Eurasian Blackbird

Oriental Magpie-Robin

White-rumped Shama

Indian Robin

Black Redstart

Common Stonechat

Pied Bushchat

Timaliinae

B Spotted Babbler Indian Scimitar-Babbler

Yellow-eyed Babbler

Common Babbler

Jungle Babbler
Scientific name

Status

Ceryle rudis (Linnaeus, 1758)

Merops orientalis Latham, 1801

SV

Coracias benghalensis (Linnaeus, 1758)

Upupa epops Linnaeus, 1758

LM

Megalaima zeylanica (Gmelin, 1788)

Megalaima haemacephala (P.L.S. Müller, 1776) B

Picumnus innominatus Burton, 1836

LM

Dendrocopos nanus (Vigors, 1832)

Dendrocopos mahrattensis (Latham, 1801) B

Celeus brachyurus (Vieillot, 1818)

பM

Dinopium benghalense (Linnaeus, 1758)

Pitta brachyura (Linnaeus, 1766)

PM

Ammomanes phoenicurus (Franklin, 1831) B

Hirundo daurica Linnaeus, 1771

B

Motacilla maderaspatensis Gmelin, 1789

Motacilla flava Linnaeus, 1758

Motacilla cinerea Tunstall, 1771

Anthus rufulus Vieillot, 1818

Anthus trivialis (Linnaeus, 1758)

WV

WV

$B$

Coracina macei (Lesson, 1830)

Coracina melanoptera (Rüppell, 1839) B

Pericrocotus cinnamomeus (Linnaeus, 1766) B

Pericrocotus erythropygius (Jerdon, 1840) LM

Pericrocotus flammeus (Forster, 1781) LM

Tephrodornis pondicerianus (Gmelin, 1789) B

Pycnonotus melanicterus (Gmelin, 1789) B

Pycnonotus jocosus (Linnaeus, 1758) B

Pycnonotus cafer (Linnaeus, 1766)

Aegithina tiphia (Linnaeus, 1758)

Lanius cristatus Linnaeus, 1758

Lanius vittatus Valenciennes, 1826

Lanius schach Linnaeus, 1758

WV

WV

Monticola cinclorhynchus (Vigors, 1832) WV

Monticola solitarius (Linnaeus, 1758) WV

Zoothera citrina (Latham, 1790) WV

Turdus unicolor Tickell, 1833

Turdus merula Linnaeus, 1758

Copsychus saularis (Linnaeus, 1758)

Copsychus malabaricus (Scopoli, 1786)

Saxicoloides fulicata (Linnaeus, 1776)

Phoenicurus ochruros (Gmelin, 1774)

Saxicola torquata (Linnaeus, 1766)

Saxicola caprata (Linnaeus, 1766)

Pellorneum ruficeps Swainson, 1832

Pomatorhinus horsfieldii Sykes, 1832

Chrysomma sinense (Gmelin, 1789)

Turdoides caudatus (Dumont, 1823)

Turdoides striatus (Dumont, 1823)
WV

B

WV

WV

B

B

B

White-breasted Kingfisher Halcyon smyrnensis(Linnaeus, 1758)

B 


\begin{tabular}{|c|c|c|}
\hline Common name & Scientific name & Status \\
\hline \multicolumn{3}{|l|}{ Sylviinae } \\
\hline Franklin's Prinia & Prinia hodgsonii Blyth, 1844 & B \\
\hline Jungle Prinia & Prinia sylvatica Jerdon, 1840 & B \\
\hline Ashy Prinia & Prinia socialis Sykes, 1832 & B \\
\hline Plain Prinia & Prinia inornata Sykes, 1832 & B \\
\hline Blyth's Reed-Warbler & Acrocephalus dumetorum Blyth, 1849 & WV \\
\hline Booted Warbler & Hippolais caligata (Lichtenstein, 1823) & WV \\
\hline Common Tailorbird & Orthotomus sutorius (Pennant, 1769) & B \\
\hline Hume's Warbler & Phylloscopus humei (Brooks, 1878) & WV \\
\hline Greenish Leaf-Warbler & Phylloscopus trochiloides (Sundevall, 1837) & 7) $\mathrm{B}$ \\
\hline Common Lesser Whitethroat & Sylvia curruca (Linnaeus, 1758) & WV \\
\hline \multicolumn{3}{|l|}{ Muscicapinae } \\
\hline Asian Brown Flycatcher & Muscicapa dauurica Pallas, 1811 & WV \\
\hline Ultramarine Flycatcher & Ficedula superciliaris (Jerdon, 1840) & WV \\
\hline Verditer Flycatcher & Eumyias thalassina (Swainson, 1838) & WV \\
\hline Tickell's Blue-Flycatcher & Cyornis tickelliae Blyth, 1843 & B \\
\hline Grey-headed Flycatcher & Culicicapa ceylonensis (Swainson, 1820) & B \\
\hline \multicolumn{3}{|l|}{ Monarchinae } \\
\hline $\begin{array}{l}\text { Black-naped } \\
\text { Monarch-Flycatcher }\end{array}$ & Hypothymis azurea (Boddaert, 1783) & B \\
\hline \multicolumn{3}{|l|}{ Rhipidurinae } \\
\hline White-browed & Rhipidura aureola Lesson, 1830 & B \\
\hline Fantail-Flycatcher & & \\
\hline \multicolumn{3}{|l|}{ Paridae } \\
\hline Black-lored Yellow Tit & Parus xanthogenys Vigors, 1831 & B \\
\hline \multicolumn{3}{|l|}{ Sittidae } \\
\hline Chestnut-bellied Nuthatch & Sitta castanea Lesson, 1830 & B \\
\hline Velvet-fronted Nuthatch & Sitta frontalis Swainson, 1820 & B \\
\hline \multicolumn{3}{|l|}{ Dicaeidae } \\
\hline Tickell's Flowerpecker & Dicaeum erythrorhynchos (Latham, 1790) & B \\
\hline \multicolumn{3}{|l|}{ Nectariniidae } \\
\hline Purple-rumped Sunbird & Nectarinia zeylonica (Linnaeus, 1766) & B \\
\hline Purple Sunbird & Nectarinia asiatica (Latham, 1790) & B \\
\hline \multicolumn{3}{|l|}{ Zosteropidae } \\
\hline Oriental White-eye & Zosterops palpebrosus (Temminck, 1824) & B \\
\hline \multicolumn{3}{|l|}{ Fringillidae } \\
\hline Common Rosefinch & Carpodacus erythrinus (Pallas, 1770) & WV \\
\hline \multicolumn{3}{|l|}{ Estrildidae } \\
\hline White-rumped Munia & Lonchura striata (Linnaeus, 1766) & B \\
\hline Black-headed Munia & Lonchura malacca (Linnaeus, 1766) & B \\
\hline \multicolumn{3}{|l|}{ Passerinae } \\
\hline House Sparrow & Passer domesticus (Linnaeus, 1758) & B \\
\hline \multicolumn{3}{|l|}{ Sturnidae } \\
\hline Brahminy Starling & Sturnus pagodarum (Gmelin, 1789) & B \\
\hline Asian Pied Starling & Sturnus contra Linnaeus, 1758 & B \\
\hline Common Myna & Acridotheres tristis (Linnaeus, 1766) & B \\
\hline Bank Myna & Acridotheres ginginianus (Latham, 1790) & LM \\
\hline \multicolumn{3}{|l|}{ Dicruridae } \\
\hline Black Drongo & Dicrurus macrocercus Vieillot, 1817 & B \\
\hline Ashy Drongo & Dicrurus leucophaeus Vieillot, 1817 & WV \\
\hline White-bellied Drongo & Dicrurus caerulescens (Linnaeus, 1758) & B \\
\hline Bronzed Drongo & Dicrurus aeneus Vieillot, 1817 & B \\
\hline \multicolumn{3}{|l|}{ Corvidae } \\
\hline Indian Treepie & Dendrocitta vagabunda (Latham, 1790) & B \\
\hline Grey Treepie & Dendrocitta formosae Swinhoe, 1863 & LM \\
\hline House Crow & Corvus splendens Vieillot, 1817 & B \\
\hline Jungle Crow & Corvus macrorhynchos Wagler, 1827 & B \\
\hline
\end{tabular}

$\mathrm{B}=$ Breeding birds or Residents, $\mathrm{WV}=$ Winter Visitor, $\mathrm{LM}=$ Local Migrant, $\mathrm{PM}$ = Passage Migrant, $\mathrm{SV}=$ Summer Visitor

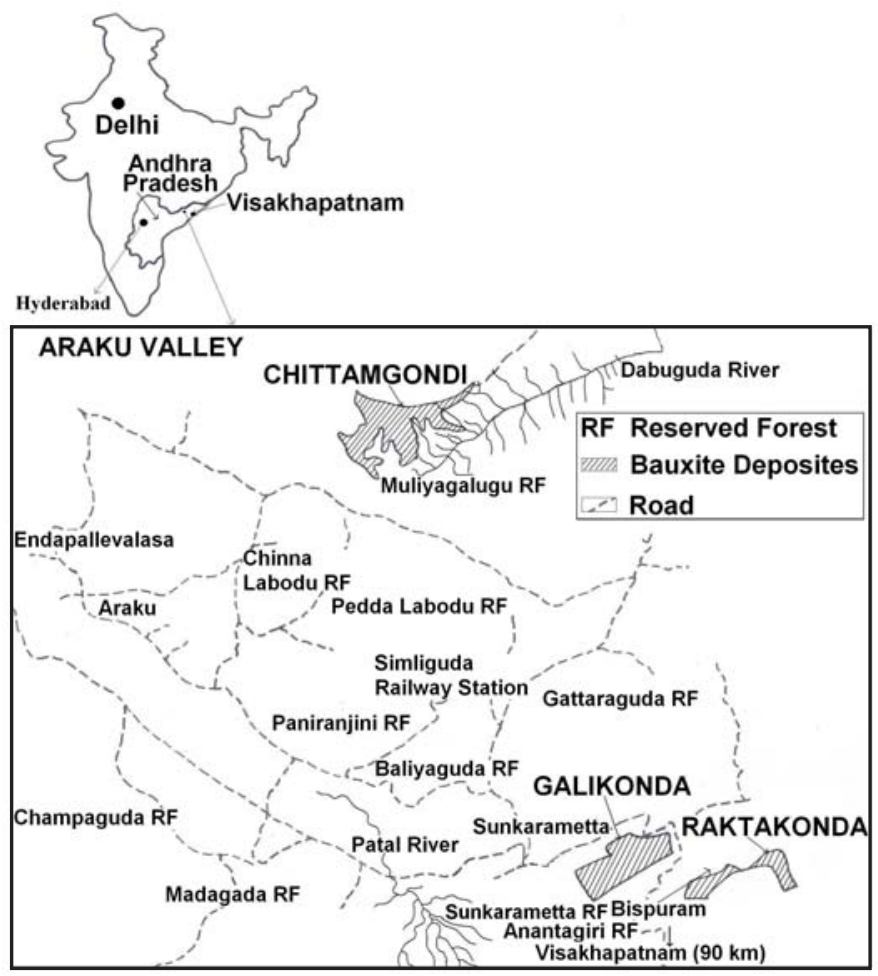

Figure 1. Study area map

Table 2. List of globally threatened birds observed in Araku Valley

\begin{tabular}{lll}
\hline Common name & Scientific name & BLI catogory \\
\hline Purple Wood-Pigeon & Columba punicea Blyth, 1842 & Vulnerable \\
Pallid Harrier & Circus macrourus (S.G. Gmelin, 1770) & Near Threatened \\
Greater Spotted Eagle & Aquila clanga Pallas, 1811 & Vulnerable \\
Lesser Kestrel & Falco naumanni Fleischer, 1818 & Vulnerable \\
\hline
\end{tabular}

BirdLife International (2001)

Common Hoopoe Upupa epops, Plain Prinia Prinia inornata and White-rumped Shama Copsychus malabaricus were commonly nesting in the scrub jungle and wooded forests. As many as 112 species of birds in Araku Valley are resident breeders, 23 species winter visitors, 09 species local migrants, two species passage migrants and one species (Small Beeeater Merops orientalis) was summer visitor. Most of the bird species use more than one habitat for their activities such as nesting, roosting and foraging. Fifty-five species were sighted in the agriculture mixed urban area, 54 species in plantation, 114 species in scrub jungle, 63 species in well wooded forest and 31 species in the Phoenix dominated areas. However, these habitats were not exclusive to the species.

\section{Discussion and Conclusions}

The animal diversity of the Araku Valley region is facing serious threats due to habitat loss and habitat degradation. Three mammalian species: Leopard Panthera pardus, Sloth Bear Melursus ursinus and Indian Giant Squirrel Ratufa indica under Schedule I of the Indian Wildlife Protection Act (1972) inhabit the area. Podu cultivation, fuel wood collection, customary hunting, forest fire and cutting trees are amongst threats from the locals. However, mining is a much more serious issue. Activities including opencast mining that 
adversely affect the ecosystem are likely to negatively impact the avifauna of the region. Large scale mining for bauxite is proposed almost all along the Eastern Ghats by various organizations. Vehement protests by the locals are also on against many such projects. Hunting, shifting cultivation, pastoralism, and settled cultivation are common practices among the indigenous people of the Eastern Ghats in deriving livelihood from the local environment. Earlier the people here followed a shifting cultivation system with a longer cycle, leaving the land untouched and fallow for longer periods to recoup. But with gradual increase in population, podu cultivation with shorter cycles has led to drastic loss of natural resources, causing serious erosion of the surface soil, particularly in the slopes, repeated with short-period cycles, the podu cultivation has serious long-standing impacts. As a common ritual during April, the whole Araku Valley tribes celebrate hunting festival, during which many species are hunted.

\section{References}

Abdulali, H. (1945). Birds of the Vizagapatnam District. Journal of the Bombay Natural History Society 45: 333-347.

Abdulali, H. (1953). More about Vizagapatnam birds. Journal of Bombay Natural History Society 51: 746-747.

Ali, S. \& S.D. Ripley (1969). Handbook of the Birds of India and Pakistan together with those of Nepal, Sikkim, Bhutan and Ceylon, 3. Stone Curlews to Owls. Oxford University Press, Bombay, xiv+327pp.

Ali, S. \& S.D. Ripley (1983). Handbook of the Birds of India and Pakistan. Oxford University Press, Oxford, xiii+737pp.

Azeez, P.A., S. Bhupathy, S.N. Prasad, R. Chandra \& T. Selva Kumar (2008). Report of the Status of Blewitt's Owl in Araku Valley and Environmental Management Plan in view of the Proposed Bauxite Mines. Sálim Ali Centre for Ornithology and Natural History, Coimbatore, India, 89pp.

Bibby, C.J., N.D. Burgess \& D.A. Hill (1993). Bird Census Techniques. Academic Press Limited, London, xvii+257pp.

BirdLife International (2001). Threatened Birds of Asia - The Birdlife International Red Data Book. Birdlife International, Cambridge, UK, $x x x+3038 p p$.
Champion, H.G. \& S.K. Seth (1968). A Revised Survey of the Forest Types of India. Government of India, New Delhi, xxvii+404pp.

Chandra, R., B.A.K. Prusty, D. Mythily, S.R. Sarimol \& P.A. Azeez (2007). Nutrients and alkali metal distribution in the top soil of bauxite rich hillocks in Araku Valley, Andhra Pradesh, India. Environmental Science: An Indian Journal 2(3): 145-153.

Grimmett, R., C. Inskipp \& T. Inskipp (1999). Birds of the Indian Subcontinent. Cristopher Helm Publishers, Oxford University Press, 384pp.

Manakadan, R. \& A. Pittie (2004). Standardized common and scientific names of the birds of the Indian subcontinent. Buceros Envis Newsletter. Avian Ecology \& Indian Wetlands 6(1): 1-37.

Price, D.T. (1978). Some observations on the warbler population of the upland perennial wetlands in the Eastern Ghats. Journal of Bombay Natural History Society 75(2): 488-490.

Price, D.T. (1979). The seasonality and occurrence of birds in the eastern Ghats of Andhra Pradesh. Journal of Bombay Natural History Society 76(3): 379-422.

Price, D.T. (1990). The impact of loss of forest on the birds of the eastern Ghats of Andhra Pradesh, pp. 549-606. In: Daniel, J.C \& J.S. Serrao (eds.). Conservation in Developing Countries: Problems and Prospects. Proceedings of the centenary seminar of Bombay Natural History Society, Bombay. Oxford University Press.

Raju, K.S.R. \& D.T. Price (1973). Tree sparrow Passer montanus (L.) in the Eastern Ghats. Journal of Bombay Natural History Society 70(3): 557-558.

Raju, K. \& J.P. Selvin (1971). Little spiderhunter. Arachnothera longirostris (Latham) in the Eastern Ghats. Ibis 68: 454.

Ripley, S.D., B.M. Beehler \& K.S.R.K. Raju (1987-88). Birds of Visakhapatnam Ghats, Andhra Pradesh, (Parts 1 \& 2). Journal of Bombay Natural History Society 84(3) \& 85(1): 540-559, 90-107.

Srinivasulu, C. (2004). Birds of Kawal Sanctuary, Andhra Pradesh. Journal of Bombay Natural History Society 101(1): 3-25.

Srinivasulu, C. (2006). Additions to the mammalian and avian diversity of Nallamala Hills, with two mammal records. Zoos' Print Journal 21(3): 2185-2186.

Whistler, H. \& N.B. Kinnear (1930-37). The vernay scientific survey of the eastern Ghats-Ornithological Section (16 parts). Journal of Bombay Natural History Society 36-39: 720-735; 505-524; 67-93; 96-105; 418-437. 